\title{
A Deterministic Polynomial-time Approximation Scheme for Counting Knapsack Solutions
}

\author{
Daniel Stefankovic* ${ }^{*}$ Santosh Vempala ${ }^{\dagger} \quad$ Eric Vigoda $^{\dagger}$
}

October 30, 2018

\begin{abstract}
Given $n$ elements with nonnegative integer weights $w_{1}, \ldots, w_{n}$ and an integer capacity $C$, we consider the counting version of the classic knapsack problem: find the number of distinct subsets whose weights add up to at most the given capacity. We give a deterministic algorithm that estimates the number of solutions to within relative error $1 \pm \varepsilon$ in time polynomial in $n$ and $1 / \varepsilon$ (fully polynomial approximation scheme). More precisely, our algorithm takes time $O\left(n^{3} \varepsilon^{-1} \log (n / \varepsilon)\right)$. Our algorithm is based on dynamic programming. Previously, randomized polynomial time approximation schemes were known first by Morris and Sinclair via Markov chain Monte Carlo techniques, and subsequently by Dyer via dynamic programming and rejection sampling.
\end{abstract}

\section{Introduction}

Randomized algorithms are usually simpler and faster than their deterministic counterparts. In spite of this, it is widely believed that $\mathrm{P}=\mathrm{BPP}$ (see, e.g., [2]), i.e., at least up to polynomial complexity, randomness is not essential. This conjecture is supported by the fact that there are relatively few problems for which exact randomized polynomial-time algorithms exist but deterministic ones are not known. Notable among them is the problem

${ }^{*}$ Department of Computer Science, University of Rochester, Rochester, NY 14627. Email: stefanko@cs.rochester.edu. Research supported in part by NSF grant CCF0910415.

${ }^{\dagger}$ School of Computer Science, Georgia Institute of Technology, Atlanta GA 30332. Email: \{vempala,vigoda\}@cc.gatech.edu. Research supported in part by NSF grant CCF0830298 and CCF-0910584. 
of testing whether a polynomial is identically zero (a special case of this, primality testing was open for decades but a deterministic algorithm is now known, [1]).

However, when one moves to approximation algorithms, there are many more such examples. The entire field of approximate counting is based on Markov chain Monte Carlo (MCMC) sampling [11, a technique that is inherently randomized, and has had remarkable success. The problems of counting matchings [9, 12], colorings [8], various tilings, partitions and arrangements [14], estimating partition functions [10, 16], or volumes [6, 13. are all solved by first designing a random sampling method and then reducing counting to repeated sampling. In all these cases, when the input is presented explicitly, it is conceivable that deterministic polynomial-time algorithms exist 1

The one notable example of a deterministic approximate counting algorithm is Weitz's algorithm [17] for counting independent sets weighted by an activity $\lambda$ for graphs of maximum degree $\Delta$ when $\Delta$ is constant and $\lambda<\lambda_{u}(\Delta)$ where $\lambda_{u}(\Delta)$ is the uniqueness threshold for the $\Delta$-regular tree. This was later extended to counting all matchings of bounded degree graphs 44. An alternative deterministic approach of Bandyopadhyay and Gamarnik [3] for colorings and independent sets of bounded degree graphs only approximates the logarithm of the size of the feasible set. The results of [17, 4] are the only two examples of an FPAS (fully polynomial approximation scheme) for a \#P-complete problem that we are aware of. One limitation of both of these results is that the running time is quite large, in particular, the exponent depends on $\ln \Delta$. In contrast, our algorithm has a small polynomial running time.

Here we consider one of the most basic counting problems, namely approximately counting the number of $0 / 1$ knapsack solutions. More precisely, we are given a list of nonnegative integer weights $w_{1}, \ldots, w_{n}$ and an integer capacity $C, 2$ and wish to count the number of subsets of the weights that add up to at most $C$. This decision version of this problem is NP-hard, but has a well-known pseudo-polynomial algorithm based on dynamic programming. For any $\varepsilon>0$, we give a deterministic algorithm that estimates the number of solutions to within relative error $\varepsilon$ in time polynomial in $n$ and $1 / \varepsilon$.

Our result follows a line of work in the literature. Dyer et al. [7] gave a

\footnotetext{
${ }^{1}$ Volume computation has an exponential lower bound for deterministic algorithms, but that is due to the more general oracle model in which the input is presented.

${ }^{2}$ Our results extend to real-valued inputs, but we do not consider that here to avoid the issue of the model of computation.
} 
randomized subexponential time algorithm for this problem, based on nearuniform sampling of feasible solutions by a random walk. Morris and Sinclair [15] improved this, showing a rapidly mixing Markov chain, and obtained an FPRAS (fully polynomial randomized approximation scheme). The proof of convergence of the Markov chain is based on the technique of canonical paths and a notion of balanced permutations introduced in their analysis. In a surprising development, Dyer [5], gave a completely different approach, combining dynamic programming with simple rejection sampling to also obtain an FPRAS. Although much simpler, randomization still appears to be essential in his approach - without the sampling part, his algorithm only gives a factor $n$ approximation.

Our algorithm is also based on dynamic programming, and similar to Dyer, is inspired by the pseudo-polynomial algorithm for the decision/optimization version of the knapsack problem. The complexity of the latter algorithm is $O(n C)$, where $C$ is the capacity bound. A similar complexity can be achieved for the counting problem as well using the following recurrence:

$$
S(i, j)=S(i-1, j)+S\left(i-1, j-w_{i}\right)
$$

with appropriate initial conditions. Here $S(i, j)$ is the number of knapsack solutions that use a subset of the items $\{1, \ldots, i\}$ and their weights sum to at most $j$.

Roughly speaking, since we are only interested in approximate counting, Dyer's idea was the following: scale down the capacity to a polynomial in $n$, scale down the weights by the same factor and round down the new weights, and then count the solutions to the new problem efficiently using the pseudopolynomial time dynamic programming algorithm. The new problem could have more solutions (since we rounded down) but Dyer showed it has at most a factor of $n$ more for a suitable choice of scaling. Further, given the exact counting algorithm for the new problem, one gets an efficient sampler, then uses rejection sampling to only sample solutions to the original problem. The sampler leads to a counting algorithm using standard techniques. Dyer's algorithm has running time $O\left(n^{3}+\varepsilon^{-2} n^{2}\right)$ using the above approach, and $O\left(n^{2.5} \sqrt{\log \left(\varepsilon^{-1}\right)}+n^{2} \varepsilon^{-2}\right)$ using a more sophisticated approach that also utilizes randomized rounding.

To remove the use of randomness, one might attempt to use a more coarse-grained dynamic program, namely rather than consider all integer capacities $1,2, \ldots, C$, what if we only consider weights that go up in some geometric series? This would allow us to reduce the table size to $n \log C$ rather than $n C$. The problem is that varying the capacity even by an ex- 
ponentially small factor $\left(1+n / 2^{n}\right)$ can change the number of solutions by a constant factor!

Instead, we index the table by the prefix of items allowed and the number of solutions, with the entry in the table being the minimum capacity that allows these indices to be feasible. We can now consider approximate numbers of solutions and obtain a small table.

Our main result is the following:

Theorem 1.1. Let $w_{1}, \ldots, w_{n}$ and $C$ be an instance of a knapsack problem. Let $Z$ be the number of solutions of the knapsack problem. There is a deterministic algorithm which for any $\varepsilon \in(0,1)$ outputs $Z^{\prime}$ such that $(1-\varepsilon) Z \leq Z^{\prime} \leq Z$. The algorithm runs in time $O\left(n^{3} \varepsilon^{-1} \log (n / \varepsilon)\right)$.

The running time of our algorithm is competitive with that of Dyer. One interesting improvement is the dependence on $\epsilon$. Our algorithm has a linear dependence on $\epsilon^{-1}$ (ignoring the logarithm term), whereas Monte Carlo approaches, including Dyer's algorithm [5] and earlier algorithms for this problem [15, 7], have running time which depends on $\varepsilon^{-2}$.

\section{Algorithm}

In this section we present our dynamic programming algorithm. Fix an knapsack instance and fix an ordering on the elements and their weights.

We begin by defining the function $\tau:\{0, \ldots, n\} \times \mathbb{R}_{\geq 0} \rightarrow \mathbb{R} \cup\{ \pm \infty\}$ where $\tau(i, a)$ is the smallest $C$ such that there exist at least $a$ solutions to the knapsack problem with weights $w_{1}, \ldots, w_{i}$ and capacity $C$. We can not compute the function $\tau$ efficiently since the second argument ranges over all real numbers. It will be used in the analysis and it is useful for motivating the definition of our algorithm.

Note that, by definition, $\tau(i, a)$ is monotone in $a$, that is,

$$
a \leq a^{\prime} \Longrightarrow \tau(i, a) \leq \tau\left(i, a^{\prime}\right) .
$$

The value of $\tau$ is easy to compute for $i=0$ :

$$
\tau(0, a)=\left\{\begin{aligned}
-\infty & \text { if } a=0 \\
0 & \text { if } 0<a \leq 1 \\
\infty & \text { otherwise }
\end{aligned}\right.
$$

Note that the number of knapsack solutions satisfies:

$$
Z=\max \{a: \tau(n, a) \leq C\} .
$$

We will show that $\tau(i, a)$ satisfies the following recurrence. 
Lemma 2.1. For any $i \in[n]$ and any $a \in \mathbb{R}_{\geq 0}$ we have

$$
\tau(i, a)=\min _{\alpha \in[0,1]} \max \left\{\begin{array}{l}
\tau(i-1, \alpha a), \\
\tau(i-1,(1-\alpha) a)+w_{i} .
\end{array}\right.
$$

We defer the proof of the above lemma to Section 3 ,

Now we move to an approximation of $\tau$ that we can compute efficiently. We define a function $T$ which only considers a small set of values $a$ for the second argument in the function $\tau$, these values will form a geometric progression.

Let

$$
Q:=1+\frac{\varepsilon}{n+1}
$$

and let

$$
s:=\left\lceil n \log _{Q} 2\right\rceil .
$$

The function $T:\{0, \ldots, n\} \times\{0, \ldots, s\} \rightarrow \mathbb{R}_{\geq 0} \cup\{\infty\}$ is defined using the recurrence (4) that the function $\tau$ satisfies. Namely, $T$ is defined by the following recurrence:

$$
T[i, j]=\min _{\alpha \in[0,1]} \max \left\{\begin{array}{l}
T\left[i-1,\left\lfloor j+\ln _{Q} \alpha\right\rfloor\right] \\
T\left[i-1,\left\lfloor j+\ln _{Q}(1-\alpha)\right\rfloor\right]+w_{i} .
\end{array}\right.
$$

More precisely, $T$ is defined by the following algorithm CountKnapsack. 


\section{CountKnapsack}

Input: Integers $w_{1}, w_{2}, \ldots, w_{n}, C$ and $\varepsilon>0$.

1. Set $T[0,0]=0$ and $T[0, j]=\infty$ for $j>0$.

2. Set $Q=(1+\varepsilon /(n+1))$ and $s=\left\lceil n \log _{Q} 2\right\rceil$.

3. For $i=1 \rightarrow n$, for $j=0 \rightarrow s$, set

$$
T[i, j]=\min _{\alpha \in[0,1]} \max \left\{\begin{array}{l}
T\left[i-1,\left\lfloor j+\ln _{Q} \alpha\right\rfloor\right] \\
T\left[i-1,\left\lfloor j+\ln _{Q}(1-\alpha)\right\rfloor\right]+w_{i},
\end{array}\right.
$$

where, by convention, $T[i-1, k]=0$ for $k<0$.

4. Let

$$
j^{\prime}:=\max \{j: T[n, j] \leq C\} .
$$

5. Output $Z^{\prime}:=Q^{j^{\prime}+1}$.

The minimum in the recurrence (5), although formally over the entire interval $[0,1]$, only needs to be evaluated at the discrete subset where the second argument goes to the next integer. Hence we will be able to compute $T$ efficiently.

The key fact is that $T$ approximates $\tau$ in the following sense.

Lemma 2.2. Let $i \geq 1$. Assume that for all $j \in\{0, \ldots, s\}$ we have that $T[i-$ $1, j]$ satisfy (66). Then for all $j \in\{0, \ldots, s\}$ we have that $T[i, j]$ computed using (5) satisfies:

$$
\tau\left(i, Q^{j-i}\right) \leq T[i, j] \leq \tau\left(i, Q^{j}\right)
$$

We defer the proof of Lemma 2.2 to Section 3 .

We can now prove that the output $Z^{\prime}$ of the algorithm CountKnapsack is a $(1 \pm \varepsilon)$ multiplicative approximation of $Z$.

Note that $Z^{\prime}$ is never an underestimate of $Z$, since,

$$
C<T\left[n, j^{\prime}+1\right] \leq \tau\left(n, Q^{j^{\prime}+1}\right),
$$

that is, there are at most $Q^{j^{\prime}+1}$ solutions. We also have

$$
\tau\left(n, Q^{j^{\prime}-n}\right) \leq T\left[n, j^{\prime}\right] \leq C,
$$


that is, there are at least $Q^{j^{\prime}-n}$ solutions. Hence

$$
\frac{Z^{\prime}}{Z} \leq \frac{Q^{j^{\prime}+1}}{Q^{j^{\prime}-n}}=Q^{n+1} \leq \mathrm{e}^{\varepsilon}
$$

This proves that the output $Z^{\prime}$ of the algorithm CountKnapsack satisfies the conclusion of Theorem 1.1. It remains to show that the algorithm can be modified to achieve the claimed running time.

\section{$2.1 \quad$ Running Time}

As noted earlier, the minimum in the recurrence (5) only needs to be evaluated at the discrete subset $S$ where the second argument goes to the next integer. For $j \in\{0,1, \ldots, s\}$, the set $S$ is $S=S_{1} \cup S_{2}$ where:

$$
S_{1}=\left\{Q^{-j}, \ldots, Q^{0}\right\} \text { and } S_{2}=\left\{1-Q^{0}, \ldots, 1-Q^{-j}\right\} .
$$

Thus, $T[i, j]$ can be computed in $O(s)$ time. Since there are $O(n s)$ entries of the table and $s=O\left(n^{2} / \varepsilon\right)$ the algorithm CountKnapsack can be implemented in $O\left(n s^{2}\right)=O\left(n^{5} / \varepsilon^{2}\right)$ time.

To improve the running time, recall that $\tau(i, a)$ is a non-decreasing function in $a$. Similarly, it is easy to see by induction that $T[i, j]$ is a nondecreasing function in $j$. Hence, in (5), the first argument in the maximum (namely, $T\left[i-1,\left\lfloor j+\ln _{Q} \alpha\right\rfloor\right]$ ) is non-decreasing in $\alpha$. Similarly, the second argument in the maximum is a non-increasing function in $\alpha$. Hence the minimum of the maximum of the two arguments occurs either at the boundary (that is, for $\alpha \in\{0,1\}$ ) or for $\alpha \in(0,1)$ where the derivative changes from negative to positive, that is $\alpha$ such that for $\beta<\alpha$

$$
T\left[i-1,\left\lfloor j+\ln _{Q} \beta\right\rfloor\right]<T\left[i-1,\left\lfloor j+\ln _{Q}(1-\beta)\right\rfloor\right]+w_{i},
$$

and for $\beta>\alpha$

$$
T\left[i-1,\left\lfloor j+\ln _{Q} \beta\right\rfloor\right] \geq T\left[i-1,\left\lfloor j+\ln _{Q}(1-\beta)\right\rfloor\right]+w_{i} .
$$

Therefore, if we had the set $S$ in sorted order, we can find the $\alpha$ that achieves the minimum in (5) using binary search in $O(\log s)$ time. We do not have $S$ in sorted order, but we do have $S_{1}$ and $S_{2}$ in sorted order. We can instead do binary search over $S_{1}$ to find the $\alpha \in S_{1}$ that achieves the minimum over that set, and then over $S_{2}$, and finally compare the two values. Therefore, step 3 of the algorithm CountKnapsack to compute $T[i, j]$ can be implemented in $O(\log s)$ time, and the entire algorithm then takes $O\left(n^{3} \varepsilon^{-1} \log (n / \varepsilon)\right)$ time. This completes the proof of the running time claimed in Theorem 1.1. 


\section{Proofs of Lemmas}

Here we present the proofs of the earlier lemmas.

We begin with the proof of Lemma 2.1 which presents the recurrence for the function $\tau(i, a)$.

Proof of Lemma 2.1. Fix any $\alpha \in[0,1]$. Let $B=\max \{\tau(i-1, \alpha a), \tau(i-$ $\left.1,(1-\alpha) a)+w_{i}\right\}$. There exist at least $\alpha a$ solutions with weights $w_{1}, \ldots, w_{i-1}$ and capacity $B \geq \tau(i-1, \alpha a)$. There exist at least $(1-\alpha) a$ solutions with weights $w_{1}, \ldots, w_{i-1}$ and capacity $B-w_{i} \geq \tau(i-1,(1-\alpha) a)$. Hence there exist at least $a$ solutions with weights $w_{1}, \ldots, w_{i}$ and capacity $B$ and thus $\tau(i, a) \leq B$. To see that we did not double count, note that the first type of solutions (of which there are at least $\alpha a$ ) has $x_{i}=0$ and the second type of solutions (of which there are at least $(1-\alpha) a$ ) has $x_{i}=1$.

We established

$$
\tau(i, a) \leq \min _{\alpha \in[0,1]} \max \left\{\begin{array}{l}
\tau(i-1, \alpha a), \\
\tau(i-1,(1-\alpha) a)+w_{i} .
\end{array}\right.
$$

Consider the solution of the knapsack problem with weights $w_{1}, \ldots, w_{i}$ and capacity $C=\tau(i, a)$ that has at least $a$ solutions. Let $\beta$ be the fraction of the solutions that do not include item $i$. Then $\tau(i-1, \beta a) \leq C, \tau(i,(1-$ $\beta) a) \leq C-w_{i}$, and hence

$$
\max \left\{\tau(i-1, \beta a), \tau(i,(1-\beta) a)+w_{i}\right\} \leq C=\tau(i, a) .
$$

We established

$$
\tau(i, a) \geq \min _{\alpha \in[0,1]} \max \left\{\begin{array}{l}
\tau(i-1, \alpha a), \\
\tau(i-1,(1-\alpha) a)+w_{i} .
\end{array}\right.
$$

Equations (77) and (8) yield (4).

We now prove Lemma 2.2 that the function $T$ approximates $\tau$.

Proof of Lemma 2.2. By the assumption of the lemma and (1) we have

$$
T\left[i-1,\left\lfloor j+\ln _{Q} \alpha\right\rfloor\right] \geq \tau\left(i-1, Q^{\left\lfloor j+\ln _{Q} \alpha\right\rfloor-(i-1)}\right) \geq \tau\left(i-1, \alpha Q^{j-i}\right) .
$$

and

$$
\begin{aligned}
T\left[i-1,\left\lfloor j+\ln _{Q}(1-\alpha)\right\rfloor\right] \geq \tau\left(i-1, Q^{\left\lfloor j+\ln _{Q}(1-\alpha)\right\rfloor-(i-1)}\right) & \\
& \geq \tau\left(i-1,(1-\alpha) Q^{j-i}\right) .
\end{aligned}
$$


Combining (9) and (10) with min and max operators we obtain

$$
\begin{gathered}
\left(\min _{\alpha \in[0,1]} \max \left\{\begin{array}{l}
T\left[i-1,\left\lfloor j+\ln _{Q} \alpha\right\rfloor\right], \\
T\left[i-1,\left\lfloor j+\ln _{Q}(1-\alpha)\right\rfloor\right]+w_{i}
\end{array}\right) \geq\right. \\
\left(\min _{\alpha \in[0,1]} \max \left\{\begin{array}{l}
\tau\left(i-1, \alpha Q^{j-i}\right), \\
\tau\left(i-1,(1-\alpha) Q^{j-i}\right)+w_{i}
\end{array}\right)=\tau\left(i, Q^{j-i}\right),\right.
\end{gathered}
$$

establishing that $T[i, j]$ computed using (15) satisfy the lower bound in (6).

By the assumption of the lemma and (1) we have

$$
T\left[i-1,\left\lfloor j+\ln _{Q} \alpha\right\rfloor\right] \leq \tau\left(i-1, Q^{\left\lfloor j+\ln _{Q} \alpha\right\rfloor}\right) \leq \tau\left(i-1, \alpha Q^{j}\right) .
$$

and

$$
T\left[i-1,\left\lfloor j+\ln _{Q}(1-\alpha)\right\rfloor\right] \leq \tau\left(i-1, Q^{\left\lfloor j+\ln _{Q}(1-\alpha)\right\rfloor}\right) \leq \tau\left(i-1,(1-\alpha) Q^{j}\right) .
$$

Combining (11) and (12) with min and max operators we obtain

$$
\begin{gathered}
\left(\min _{\alpha \in[0,1]} \max \left\{\begin{array}{l}
T\left[i-1,\left\lfloor j+\ln _{Q} \alpha\right\rfloor\right], \\
T\left[i-1,\left\lfloor j+\ln _{Q}(1-\alpha)\right\rfloor\right]+w_{i}
\end{array}\right) \leq\right. \\
\left(\min _{\alpha \in[0,1]} \max \left\{\begin{array}{l}
\tau\left(i-1, \alpha Q^{j}\right), \\
\tau\left(i-1,(1-\alpha) Q^{j}\right)+w_{i}
\end{array}\right)=\tau\left(i, Q^{j}\right),\right.
\end{gathered}
$$

establishing that $T[i, j]$ computed using (5) satisfy the upper bound in (6) .

\section{References}

[1] M. Agrawal, N. Kayal, and N. Saxena. PRIMES is in P. Ann. of Math., 160(2):781-793, 2004.

[2] S. Arora and B. Barak. Computational complexity: A Modern Approach. Cambridge University Press, Cambridge, 2009.

[3] A. Bandyopadhyay and D. Gamarnik. Counting without sampling: Asymptotics of the log-partition function for certain statistical physics models. Random Struct. Algorithms, 33(4):452-479, 2008.

[4] M. Bayati, D. Gamarnik, D. Katz, C. Nair, and P. Tetali. Simple deterministic approximation algorithms for counting matchings. In Proceedings of the 39th Annual ACM Symposium on Theory of Computing (STOC), pages 122-127. 2007. 
[5] M. Dyer. Approximate counting by dynamic programming. In Proceedings of the Thirty-Fifth Annual ACM Symposium on Theory of Computing (STOC), pages 693-699, 2003.

[6] M. Dyer, A. Frieze, and R. Kannan. A random polynomial-time algorithm for approximating the volume of convex bodies. J. Assoc. Comput. Mach., 38(1):1-17, 1991.

[7] M. Dyer, A. Frieze, R. Kannan, A. Kapoor, L. Perkovic, and U. Vazirani. A mildly exponential time algorithm for approximating the number of solutions to a multidimensional knapsack problem. Combin. Probab. Comput., 2(3):271-284, 1993.

[8] M. Jerrum. A very simple algorithm for estimating the number of $k$ colorings of a low-degree graph. Random Struct. Algorithms, 7(2):157$165,1995$.

[9] M. Jerrum and A. Sinclair. Approximating the permanent. SIAM J. Comput., 18(6):1149-1178, 1989.

[10] M. Jerrum and A. Sinclair. Polynomial-time approximation algorithms for the Ising model. SIAM J. Comput., 22(5):1087-1116, 1993.

[11] M. Jerrum and A. Sinclair. The Markov Chain Monte Carlo Method: An Approach To Approximate Counting and Integration. In D. S. Hochbaum, editor, Approximation Algorithms for NP-hard Problems, pages 482-520. PWS Publishing, 1996.

[12] M. Jerrum, A. Sinclair, and E. Vigoda. A polynomial-time approximation algorithm for the permanent of a matrix with nonnegative entries. J. ACM, 51(4):671-697, 2004.

[13] L. Lovász and S. Vempala. Simulated annealing in convex bodies and an $O^{*}\left(n^{4}\right)$ volume algorithm. J. Comput. System Sci., 72(2):392-417, 2006 .

[14] M. Luby, D. Randall, and A. Sinclair. Markov chain algorithms for planar lattice structures. SIAM J. Comput., 31(1):167-192, 2001.

[15] B. Morris and A. Sinclair. Random walks on truncated cubes and sampling 0-1 knapsack solutions. SIAM J. Comput., 34(1):195-226, 2004. 
[16] D. Štefankovič, S. Vempala, and E. Vigoda. Adaptive simulated annealing: a near-optimal connection between sampling and counting. $J$. ACM, 56(3):1-36, 2009.

[17] D. Weitz. Counting independent sets up to the tree threshold. In Proceedings of the 38th Annual ACM Symposium on Theory of Computing (STOC), pages 140-149. 2006. 